\title{
Potential antibiofilm activity of farnesol- loaded poly(DL-lactide-co-glycolide) (PLGA) nanoparticles against Candida albicans
}

\author{
Bükay Yenice Gürsu(D)
}

\begin{abstract}
Candida species are ubiquitous fungal pathogens and are the most common causes of mucosal and invasive fungal infections in humans. Especially Candida albicans commonly resides as a commensal in the mucosal tissues of approximately half of the human population. When the balance of the normal flora is disrupted or the immune defenses are compromised, Candida species can become pathogenic, often causing recurrent disease in susceptible individuals.

The treatments available for Candida infection are commonly drug-based and can involve topical and systemic antifungal agents. However, the use of standard antifungal therapies can be limited because of toxicity, low efficacy rates, and drug resistance. Candida species ability to produce drug-resistant biofilm is an important factor in human infections, because microorganisms within biofilm benefit from various advantages over their planktonic counterparts including protection from antimicrobials and chemicals. These limitations emphasize the need to develop new and more effective antifungal agents. Natural products are attractive alternatives for this purpose due to their broad spectrum of biological activities. Farnesol is produced by many microorganisms and found in some essential oils. It has also a great attention as a quorum-sensing molecule and virulence factor. It has also antimicrobial potential due to its inhibitory effects on various bacteria and fungi. However, as it is a hydrophobic component, its solubility and biofilm inhibiting properties are limited.

To overcome these shortcomings, nanoparticle-based drug delivery systems have been successfully used. For this purpose, especially using biodegradable polymeric nanoparticles has gained increasing attention owing to their biocompatibility and minimal toxicity. Poly (DL-lactide-co-glycolide) (PLGA) is the most widely used polymer in this area. In this study, farnesol is loaded to PLGA nanoparticles (F-PLGA NPs) by emulsion evaporation method and characterized by DLS, TEM, and FT-IR analyses. Our TEM findings indicate that the sizes of F-PLGA NPs are approximately $140 \mathrm{~nm}$. The effects of F-PLGA NPs on planktonic cells and biofilm formation of C. albicans were compared with effects of farnesol alone. Farnesol inhibits the growth at a range of $53 \%$ at a concentration of $2.5 \mu \mathrm{L}$ compared to the control group. This rate is $45 \%$ for F-PLGA NPs at the same concentration. However, although farnesol amount in F-PLGA is approximately $22.5 \%$ of the total volume, the observed effect is significant. In TEM examinations, planktonic Candida cells treated with farnesol showed relatively regular ultrastructural morphology. Few membrane and wall damage and electron density in the cytoplasm were determined. In F-PLGA NP-treated cells, increased irregular cell morphology, membrane and wall damages, and large vacuoles are observed. Our SEM and XTT data suggest that F-PLGA NPs can reduce the biofilm formation at lower concentrations than farnesol (Continued on next page)
\end{abstract}

Correspondence: bukayyenice@gmail.com

Central Research Laboratory Application and Research Center (ARUM),

Eskişehir Osmangazi University, 26480, Odunpazarı, Eskişehir, Turkey

\section{Springer Open}

(c) The Author(s). 2020 Open Access This article is licensed under a Creative Commons Attribution 4.0 International License, which permits use, sharing, adaptation, distribution and reproduction in any medium or format, as long as you give appropriate credit to the original author(s) and the source, provide a link to the Creative Commons licence, and indicate if changes were made. The images or other third party material in this article are included in the article's Creative Commons licence, unless indicated otherwise in a credit line to the material. If material is not included in the article's Creative Commons licence and your intended use is not permitted by statutory regulation or exceeds the permitted use, you will need to obtain permission directly from the copyright holder. To view a copy of this licence, visit http://creativecommons.org/licenses/by/4.0/. 
(Continued from previous page)

alone 57\%, and our results showed that F-PLGA NPs are effective and biocompatible alternatives for inhibiting growth and biofilm formation of C. albicans, but detailed studies are needed.

Keywords: Biofilm, Candida, Farnesol, PLGA, Nanoparticle

\section{Introduction}

Candida species are usually yeasts that can colonize the skin and mucous membranes; however, they may cause both widespread mucosal infections and serious lifethreatening systemic diseases in immunosuppressed individuals (Laihadı et al. 2017). C. albicans is the most commonly reported agent of invasive candidiasis $(\mathrm{Oz}$ et al. 2013). In addition to virulence factors such as germ tube and pseudohyphae production, phenotypic change, phospholipase enzyme, secreted aspartyl proteinases, and the host immune system play an important role in the development of infection. Biofilm production is also another important virulence factor of Candida species, and more than $80 \%$ of human infections are estimated to be biofilm related. Cells in biofilm are much more resistant to host defense mechanisms, phagocytosis, biocides, and antibiotic treatment than their planktonics (Dag et al. 2014).

Recently, Candida infections show an increasing resistance to fluconazole and amphotericin B, and biofilmproducing Candida species are associated with a higher mortality (Rajendran et al. 2016). Existing drugs are insufficient for biofilm treatment, and they need to be administered at higher doses and more frequently. This leads to significant side effects and toxicity. In recent years, the use of natural compounds against microorganisms resistant to conventional antibiotic and antifungal therapy has been an interesting alternative. These compounds are important because of their low cost, biocompatibility, and potential antibiotic properties. Furthermore, the resistance of microorganisms to natural compounds has not been reported to date.

Farnesol is produced as a by product of the ergosterol biosynthesis pathway, and C. albicans produces high amounts of farnesol in dense cultures (Nickerson et al. 2006). The most significant effect of farnesol is that it has an inhibitory effect on yeast-hyphae transition at levels greater or equal to $300 \mu \mathrm{M}$ (Hornby et al. 2001; Costa et al. 2019). On the other hand, it is thought to be involved in the regulation of yeast cells spreading from mature biofilm surface (Lindsay et al. 2012). It is also stated that farnesol has inhibitory effects in the initial adhesion stage of biofilm formation (Cao et al. 2005). Although there is no significant effect on biofilm maturation, single cells in the mature biofilm may become susceptible to farnesol again. Farnesol destroys the cell membrane in bacteria and affects cell-membrane functions by increasing proton permeability (Jeon et al. 2011). However, due to the hydrophobic properties of farnesol, its solubility is limited and its biofilm effect is poor (Rowat et al. 2005). For example, in order to inhibit the development of oral biofilms, it should be used in high concentrations and with multiple treatment strategies (Sims et al. 2019).

In recent studies, various drugs and natural compounds are loaded on nanovectors to increase the efficacy of the active substance and reduce the amount of used antifungal. The antimicrobial activity of natural compounds combined with the unique properties of nanoparticles and functional surfaces can be obtained (Ozturk et al, 2020). Especially poly (lactide-coglicolide) (PLGA), a synthetic polymer, is a carrier with very promising results (Landis et al, 2016). It is biocompatible and biodegradable and approved by the Food and Drug Administration (FDA). PLGA is also the most widely accepted biodegradable polymer available. The aim of this study was to investigate the effects of PLGA nanoparticles arrested with farnesol on C. albicans biofilm. With the nanoparticle synthesized, it was aimed both to improve the low solubility of farnesol in water and to increase its antimicrobial and antibiofilm effectiveness on C. albicans planktonic and biofilm cells and to compare it with the nanoparticle-free activities.

\section{Materials and methods Materials}

PLGA (Mw 40,000 to $75,000 \mathrm{~g} / \mathrm{mol}$ ), polyvinyl alcohol (98\% to $99 \%$ hydrolysis degree and average $\mathrm{Mw} 30,000$ to $50,000 \mathrm{~g} / \mathrm{mol})$, and farnesol (3,7,11-Trimethyl-2,6,10dodecatrien-1-ol) (\%95) were purchased from SigmaAldrich.

\section{Microorganism}

For antimicrobial and antibiofilm activity studies, $C$. albicans ATCC 14053 standard strain was used. It was cultured on Yeast Peptone Dextrose (YPD) medium (Sigma-Aldrich) and RPMI 1640 ( with L-glutamine, Sigma-Aldrich) broth at $37^{\circ} \mathrm{C}$ for $24 \mathrm{~h}$.

\section{Synthesis of farnesol-loaded PLGA nanoparticles}

Both unloaded PLGA nanoparticles and F-PLGA NPs were formed using the emulsion evaporation method similar to the method outlined previously (Gomes et al. 2011). For organic phase constitution, $50 \mathrm{mg}$ of PLGA 
dissolved in $2 \mathrm{~mL}$ dichloromethane with or without farnesol $(22.5 \%(\mathrm{w} / \mathrm{w})$ was prepared. The aqueous phase $(20 \mathrm{~mL})$ was created with $0.3 \%(\mathrm{w} / \mathrm{v})$ polyvinyl alcohol (PVA) in nanopure water. The organic phase added drop-wise to the aqueous phase. Then, this mixture was homogenized for $2 \mathrm{~min}$ at $9500 \mathrm{rpm}$ using an Ultra Turrax T25 basic. This emulsion was sonicated in an ice bath at $2{ }^{\circ} \mathrm{C}$ and $70 \mathrm{~W}$ of energy output during $10 \mathrm{~min}$. The organic phase (dichloromethane) was removed for 20 min using a rotoryevaporator. The same procedure was used for the preparation of unloaded control nanoparticles, but farnesol was not added to the organic solvent. After synthesis, the nanoparticles were purified by dialysis to remove the excess of PVA and farnesol. The nanoparticles were ultrafiltered with $200 \mathrm{~mL}$ of water and $50 \mathrm{~mL}$ was collected. Then, samples were lyophilized and stored in a desiccator until further use (Gomes et al. 2011).

\section{Particle size and morphology characterization}

Particle size and size distribution of the nanoparticles were measured by using Zetasizer Nano Series (NanoZS). Morphological characterization was performed using transmission electron microscopy (TEM) (Hitachi HT 7800, Japan) at an accelerating voltage of $100 \mathrm{kV}$. For analysis, sample was dropped on a copper grid and was examined after it is well dried under the TEM.

\section{FT-IR spectra}

FT-IR studies were carried out on Perkin unit (PerkinElmer Spectrum Two). PLGA nanoparticles and F-PLGA NPs were thoroughly washed three times with distilled water prior to the FT-IR experiments to remove organic compounds that were not bound to the nanoparticle surfaces. The diamond ATR technique was used for obtained samples. The wavenumber sweep was 4000 to $400 \mathrm{~cm}^{-1}$ with a resolution of $0.4 \mathrm{~cm}^{-1}$.

\section{${ }^{1} \mathrm{H}$ NMR experiment}

All of the proton $\left({ }^{1} \mathrm{H}\right)$ NMR experiments were collected with JEOL ECZ $500 \mathrm{R}$ NMR spectrometer at room temperature. Proton resonance frequency was adjusted at $500.13 \mathrm{MHz}$. Deuterium dimethyl sulfoxide (d6DMSO) was used as solvent.

\section{Antifungal properties of F-PLGA NPs}

In order to determine the antifungal efficacy of the farnesol and farnesol-loaded nanoparticles, it was used to the criteria proposed by Clinical and Laboratory Standards Institute procedure (CLSI M27-A2). RPMI 1640 (bicarbonate-free) (Sigma-Aldrich, Steinheim, Germany) and SDA (Difco Laboratories, USA/France) were used as culture media. They were prepared according to the manufacturer's instructions (Wayne, 2002).
The Candida isolates from the stock medium for fresh culture production were first incubated in RPMI 1640 liquid medium and at $37^{\circ} \mathrm{C}$ for $24 \mathrm{~h}$. Samples were then taken into $5 \mathrm{~mL}$ of $0.85 \%$ saline and the suspension turbidity adjusted to 0.5 McFarland $\left(1-5 \times 10^{6}\right.$ cells $\left./ \mathrm{mL}\right)$. The prepared initial suspensions were diluted $1 / 50$ with sterile saline and then diluted 1/20 with RPMI 1640 to a concentration of $1-5 \times 10^{3}$ cells $/ \mathrm{mL}$. The broth microdilution test was performed with 96-well microplates. RPMI-1640 (Sigma, Germany) medium was used and the final concentrations were adjusted to be $2.5 \mu / \mathrm{mL}$ active ingredient in $\mathrm{vol} / \mathrm{vol}$.

After incubation at $35^{\circ} \mathrm{C}$ for $48 \mathrm{~h}$, absorbance measurements were taken with Chromate Microplate Reader 4300. The range of MIC values of farnesol were determined as the lowest concentration ranges that could reduce fungal growth compared to the positive control.

\section{Ultrastructural changes of planktonic C. albicans cells by TEM}

The ultrastructural effects of farnesol and farnesolloaded PLGA on C. albicans ATCC 14053 isolate were evaluated by TEM and as described previously (Ozturk et al., 2020). For this purpose, the $10 \mathrm{~mL}$ cell suspension was exposed to the active substance (farnesol alone and farnesol-loaded PLGA) at a concentration of $2.5 \mu / \mathrm{mL}$ and then incubated at $37^{\circ} \mathrm{C}$ for $48 \mathrm{~h}$. The control group without active substance was also included in the study. Cell suspensions were centrifuged at $5000 \mathrm{~g}$ for $15 \mathrm{~min}$ in sterile plastic centrifuge tubes and washed sequentially with PBS (phosphate-buffered saline buffer) three times (10 min each). Each sample group was prefixed in $2.5 \%$ gluteraldehyde in $0.1 \mathrm{M}$ phosphate-buffered saline (PBS) at $4{ }^{\circ} \mathrm{C}$ for $12 \mathrm{~h}$. Then, they were washed three times in PBS and post-fixed for $2 \mathrm{~h}$ in $1 \% \mathrm{OsO}_{4}$ at room temperature. Samples were dehydrated through a graded ethanol series (30-50\%, 70\%, 90\%, 96\%, and 100\%) and were polymerized at $60^{\circ} \mathrm{C}$ for $48 \mathrm{~h}$ by embedding in epoxy resin. Ultra-thin sections of $60-\mathrm{nm}$ thickness of the blocked samples were taken onto copper grids by an ultramicrotome (Leica Ultracut R). The sections were finally stained with uranyl acetate and lead citrate (Dag et al., 2012). Samples were analyzed by Hitachi HT 7800 TEM.

\section{$\mathrm{XTT}$ reduction test}

Antibiofilm activity was measured according to the method previously described by Ramage et al. 2001). Standardized cell suspensions $\left(1 \times 10^{5} \mathrm{CFU} / \mathrm{mL}\right.$ in 200 $\mu \mathrm{L}$ RPMI 1640 medium) were added 96-well flat bottomed microtiter plate. Samples were incubated at $37^{\circ} \mathrm{C}$ for $1 \mathrm{~h}$, and then, the wells were washed with PBS. RPMI medium containing active substance was added to the wells and the plates were incubated $24 \mathrm{~h}$ at $37^{\circ} \mathrm{C}$. 
Colorimetric changes according to XTT reduction test results were evaluated with a microplate reader (Chromate Microplate Reader 4300) at 492-630 nm. Thus, it was aimed to investigate the effects of active substance application before biofilm formation.

\section{Effect of the farnesol and F-PLGA on C. albicans biofilm formation by scanning electron microscopy (SEM)}

In this study, Whatman no. 1 filter papers (6-mm diameter) were used as surface and the effect of active substances on biofilm formation (pretreatment) was analyzed with SEM (Hitachi Regulus 8230) device. For this purpose, each sterile disk with impregnated C. albicans containing $1 \times 10^{5} \mathrm{CFU} /$ $\mathrm{mL}$ in RPMI 1640 medium containing the active ingredient at a concentration of $\% 1 \mathrm{v} / \mathrm{v}$ was inoculated and incubated at $37^{\circ} \mathrm{C}$ for $24 \mathrm{~h}$. Filter paper disks containing microorganism without active substances served as a control, and each assay was performed in duplicate. For scanning electron microscopic (SEM) examination, samples were prefixed in $2.5 \%$ glutaraldehyde (prepared in $0.1 \mathrm{M}$ phosphate buffer, $\mathrm{pH}$ 7.4, for $24 \mathrm{~h}$ at $4{ }^{\circ} \mathrm{C}$ ). Then, it was rinsed twice with PBS buffer ( $\mathrm{pH} 7.4$ ) and postfixation was performed with $1 \%$ osmium tetroxide for $1-2 \mathrm{~h}$ at room temperature at dark and rinsed again with PBS. Following that, the disks were dehydrated with ethyl alcohol series (30, 50, 70, 90, and 96\%) for $15 \mathrm{~min}$ and by $100 \%$ alcohol for $30 \mathrm{~min}$. Samples were dried by critical point dryer and coated with gold with a Polaron SC7620 Sputter Coater. Finally, they were examined using a scanning electron microscope (Hitachi Regulus 8230) (Dag et al. 2014).

\section{Results}

The design and synthesis of F-loaded PLGA

The illustration scheme for F-loaded PLGA is presented in Fig. 1. Synthesis procedure was performed by emulsion evaporation method.

\section{Particle size and morphology characterization}

The obtained results for the particle size, polydispersity index, and zeta potential of farnesol-loaded PLGA are shown in Fig. 2. Our TEM data show that the F-PLGA nanoparticle sizes are approximately $140 \mathrm{~nm}$. Polydispersity index value was determined as 0.463 and less than 0.5 . PDI value below 0.5 indicates that electrical conductivity is better, relatively. In addition, zeta potential value was determined as 658.5 .

\section{FT-IR spectra}

FT-IR spectra of PLGA nanoparticles and F-PLGA nanoparticles are given in Fig. 3. Peaks observed at $3364 \mathrm{~cm}^{-1}$ in the spectrum of PLGA nanoparticles and $3431 \mathrm{~cm}^{-1}$ in the spectrum of F-PLGA nanoparticles belong to ambient humidity. $\mathrm{OH}$ peak of farnesol molecule was observed at $3263 \mathrm{~cm}^{-1}$ in the F-PLGA nanoparticles. Peaks of $\mathrm{CH} 3$ groups of PLGA and FPLGA nanoparticles were observed as 2904 and 2832 $\mathrm{cm}^{-1}$, respectively. Moreover, peaks of $\mathrm{CH}_{2}$ groups of PLGA and F-PLGA nanoparticles were observed as 2944 and $2892 \mathrm{~cm}^{-1}$, respectively. The FTIR spectrum shows characteristic peaks of PLGA, for example, PLGA showed bands in the region of $1424-1505 \mathrm{~cm}^{-1}$ corresponding to $\mathrm{CC}$ ring stretching vibration. The absorption bands at 1193 and $1178 \mathrm{~cm}^{-1}$ were assigned to the $\mathrm{CO}$ band. Reduction in the intensities of some peaks was observed in the spectrum of FPLGA according to the spectrum of PLGA (Fig. 3). Characteristic bands of PLGA removed significantly in the finger print region of PLGA. As a result, a change in the intensity of peaks in the range of 1000-1500 $\mathrm{cm}^{-1}$ was observed and there was a chemical interaction between farnesol and PLGA nanoparticles.
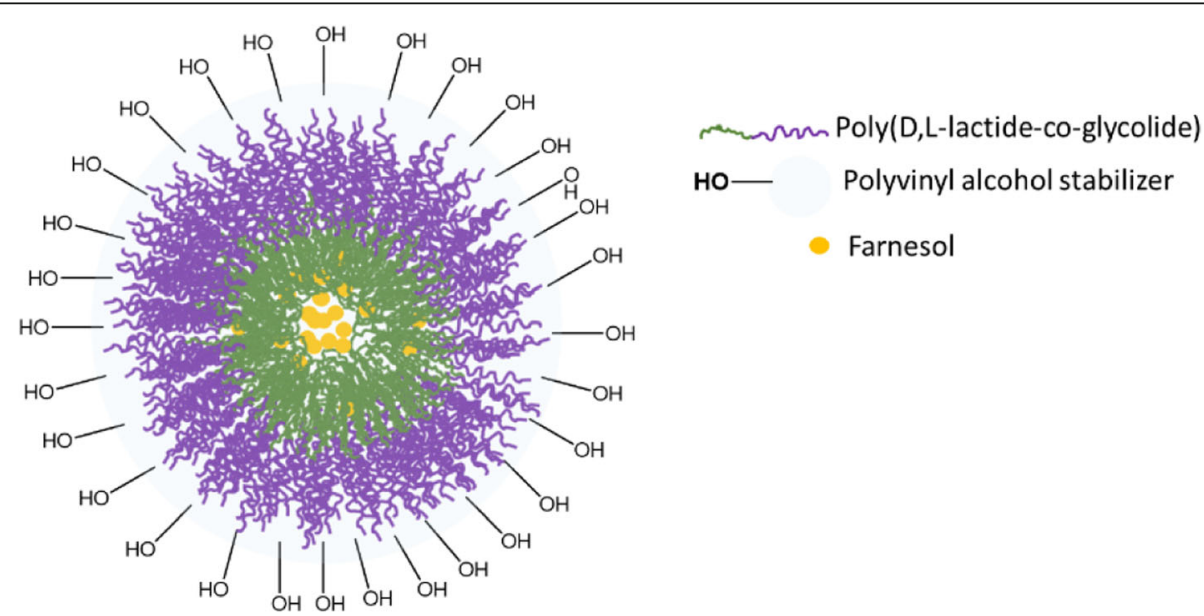

Fig. 1 Schematic illustration of farnesol-loaded particle formation 


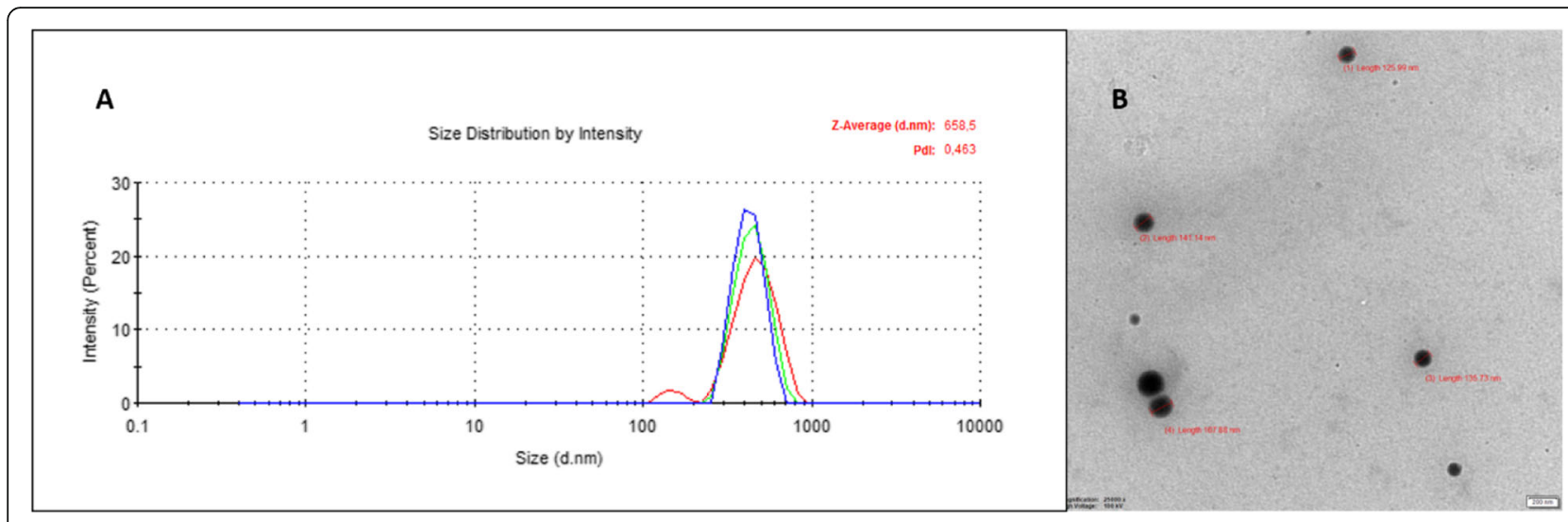

Fig. 2 Particle size, polydispersity index, and zeta potential of farnesol-loaded PLGA

\section{${ }^{1} \mathrm{H}$ NMR experiment}

${ }^{1} \mathrm{H}$ NMR spectra of farnesol and farnesol-loaded PLGA nanoparticles are given in Fig. 4. As seen in Fig. 4a, OH proton of farnesol was observed at 3.87 ppm. Moreover, methyl group's protons were seen between 1.50 and $1.60 \mathrm{ppm}$. Peaks of other protons are seen in Fig. 4a. ${ }^{1} \mathrm{H}$ NMR spectrum of farnesol-loaded PLGA nanoparticles is given in Fig. 4b. According to this spectrum, observed peaks in the range of 4-5 ppm belong to $\mathrm{OH}$ group of PVA molecule. Protons of $\mathrm{CH}_{2}$ groups of PVA molecule were seen between 1 and $2 \mathrm{ppm}$.

\section{Antifungal properties of F-PLGA NPs}

In our study, it was determined that methanol used to dissolve farnesol and used at $2.5 \%$ final concentration did not inhibit the growth of C. albicans ATCC 14053 isolate. The growth in the control group was accepted as $100 \%$, and the reduction in MIC concentration values was given as \%. According to the obtained data, farnesol

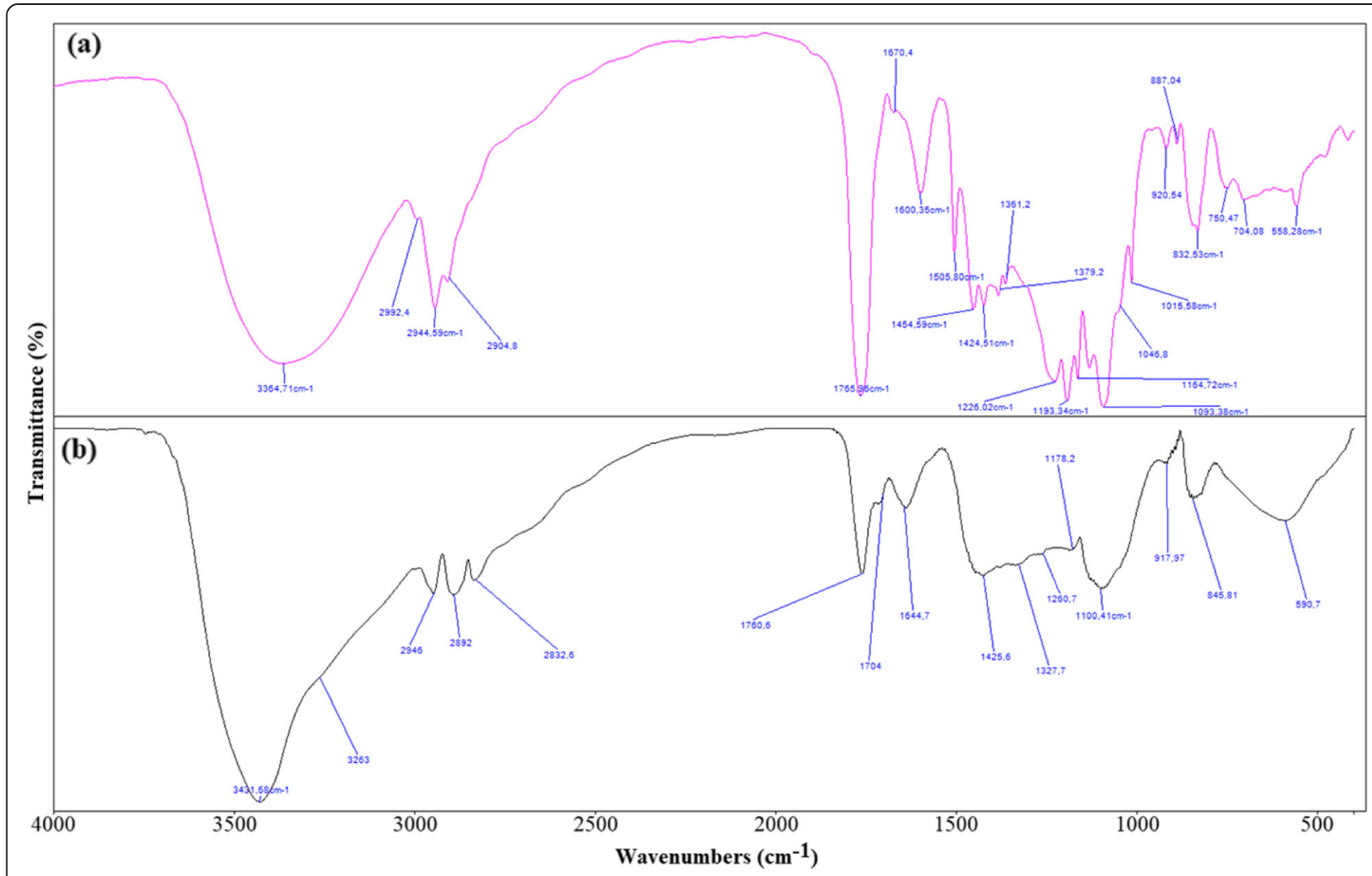

Fig. 3 FT-IR spectra of PLGA nanoparticles (a) and F-PLGA nanoparticles (b) 


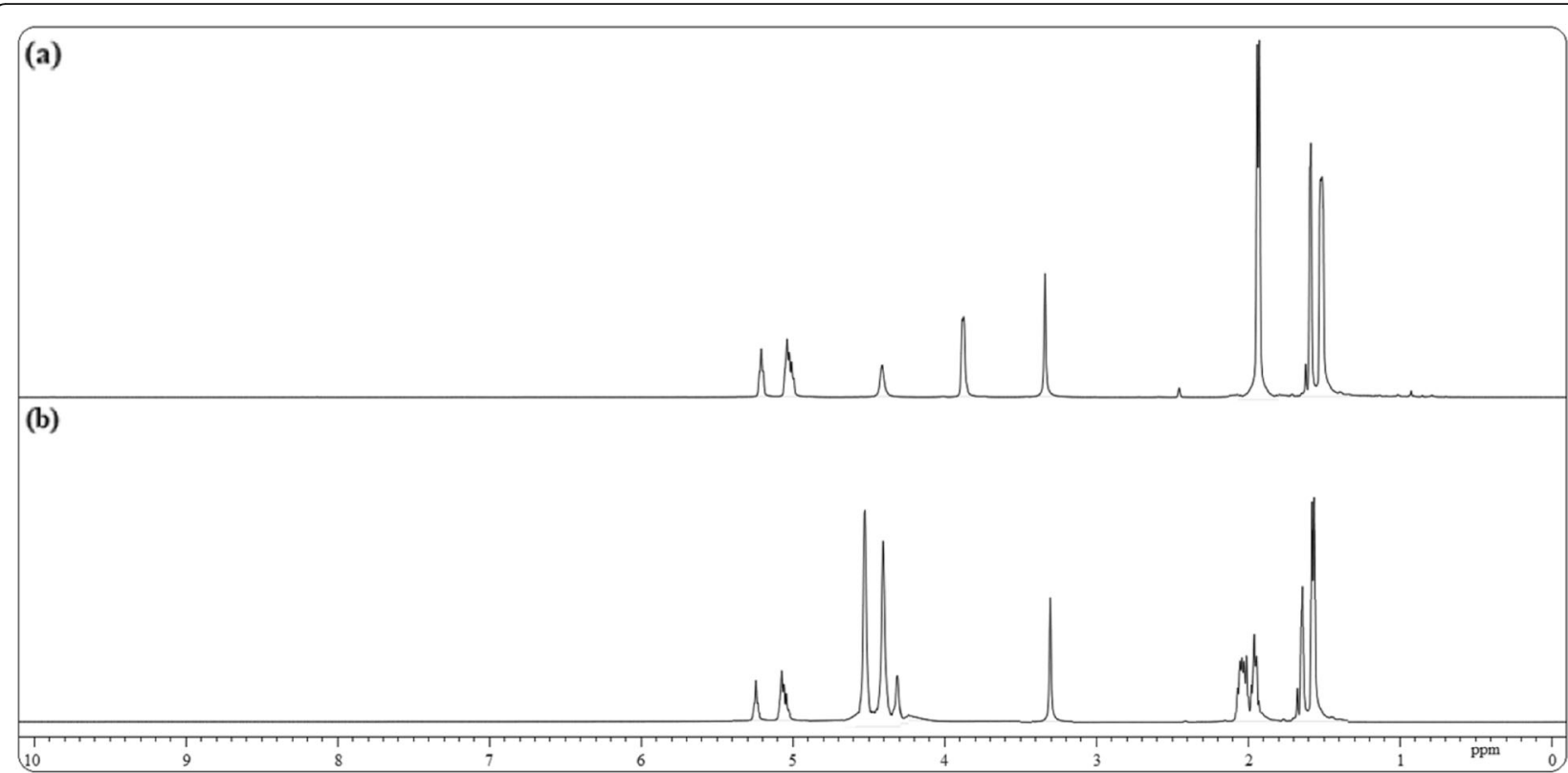

Fig. $4{ }^{1} \mathrm{H}$ NMR spectra of farnesol (a) and farnesol-loaded PLGA nanoparticles (b)

reduced the growth but not completely inhibited (53 to $6 \%)$ when used alone and at concentrations of 2.5 to $0.63 \mu \mathrm{L} / \mathrm{mL}$. Similarly, for F-PLGA, inhibition rates were also determined based on the decrease in absorbance values at concentrations of 2.5 to $1.25 \mu \mathrm{L} / \mathrm{mL}$. Reduction in growth was detected with an inhibition rate of approximately 45 to $3 \%$ (Table 1 ).

\section{Ultrastructural changes of planktonic C. albicans cells by TEM}

The effects of farnesol and F-PLGA nanoparticles on $C$. albicans ultrastructure were evaluated by TEM. As shown in Fig. 5a-c, control group cells showed wellpreserved morphological features; typical and distinct cell wall-membrane structures were present. Candida cells treated with farnesol alone showed regular wall and membrane structures and normal oval-round structure of the cell are mostly preserved but the cytoplasm is electron dense appearance. A few cells have small vacuole formation and membrane-wall damage or rupture in the cytoplasm (Fig. 5d-g).

In cells treated with F-PLGA nanoparticles, significant increases in vacuolizations were observed and they were generally quite large and numerous. In addition, increased membrane and wall damages, occasionally lysed

Table 1 MIC range values observed for farnesol and F-PLGA Nps against and Candida albicans

\begin{tabular}{lll}
\hline Parameter & Farnesol & F-PLGA Np \\
\hline MIC $v / \mathrm{V}$ & 2.5 to $0.63 \mu \mathrm{L} / \mathrm{mL}$ & 2.5 to $1.25 \mu \mathrm{L} / \mathrm{mL}$ \\
\hline
\end{tabular}

Values for $n=3 ; \mathrm{CV} \leq \% 5$ cells, and generally an irregular morphology were observed (Fig. 5h-k).

\section{Antibiofilm activity}

In this study, farnesol and F-PLGA NPs were applied to the cells at the beginning $(0 \mathrm{~h})$ of the study and the effects of application before biofilm formation (pre-treatment) were compared. The growth in the control group was accepted as $100 \%$, and results were compared accordingly. XTT reduction test data demonstrated the suppressive effect of both farnesol and F-PLGA NPs on biofilm formation compared to the control group. However, the effect of F-PLGA NPs with was much greater (53\%) (Fig. 6).

\section{SEM studies}

Figure 7 shows SEM images of the C. albicans cells treated with farnesol alone and F-PLGA NPs on biofilm formation. Untreated control group Candida cells showed the dense biofilm and EPS matrix covered on disk surface (Fig. 7a). SEM image of the biofilm treated with farnesol alone displays that the biofilm was decreased compared with the control group (Fig. 7b). As shown in Fig. 7c, there was no significant biofilm structure observed in the F-PLGA NP-treated group; only small amounts of microorganisms could be observed.

\section{Discussion}

Farnesol is an sesquiterpene alcohol found in various plant extracts and is also a QS molecule produced by $C$. albicans. It is produced endogenously as a precursor of sterol synthesis. Due to its ability to suppress hyphae 


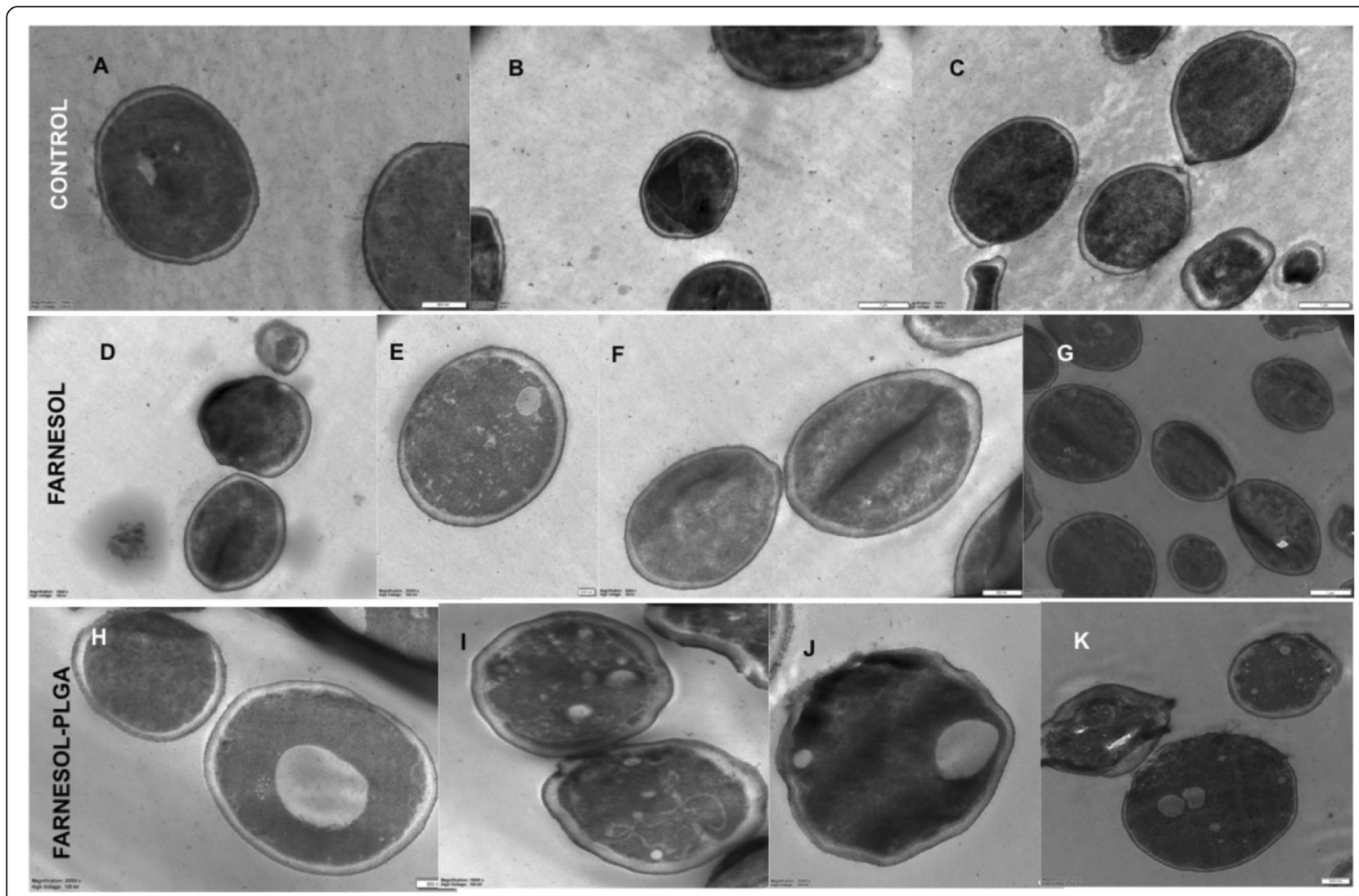

Fig. 5 TEM images of the effects of farnesol and F-PLGA nanoparticles on C. albicans ultrastructure

development in various conditions and its inhibitory effect on some pathogenic microorganisms, its use for therapeutic purposes attracts great attention (Costa et al. 2019). In the literature, it is stated that farnesol destroys the cell membranes of bacteria and thus increases the proton permeability by affecting cell-membrane functions. Farnesol is also an interesting component in terms of antibiofilm strategies. In a study by Sebaa et al., specific antibiofilm effects of farnesol and tyrosol, which are quorum-sensing molecules, were demonstrated on Candida isolates. However, this effect was independent of the fungistatic or fungicidal effect. The researchers stated that by the addition of exogenous quorumsensing molecules, the related mechanism may be

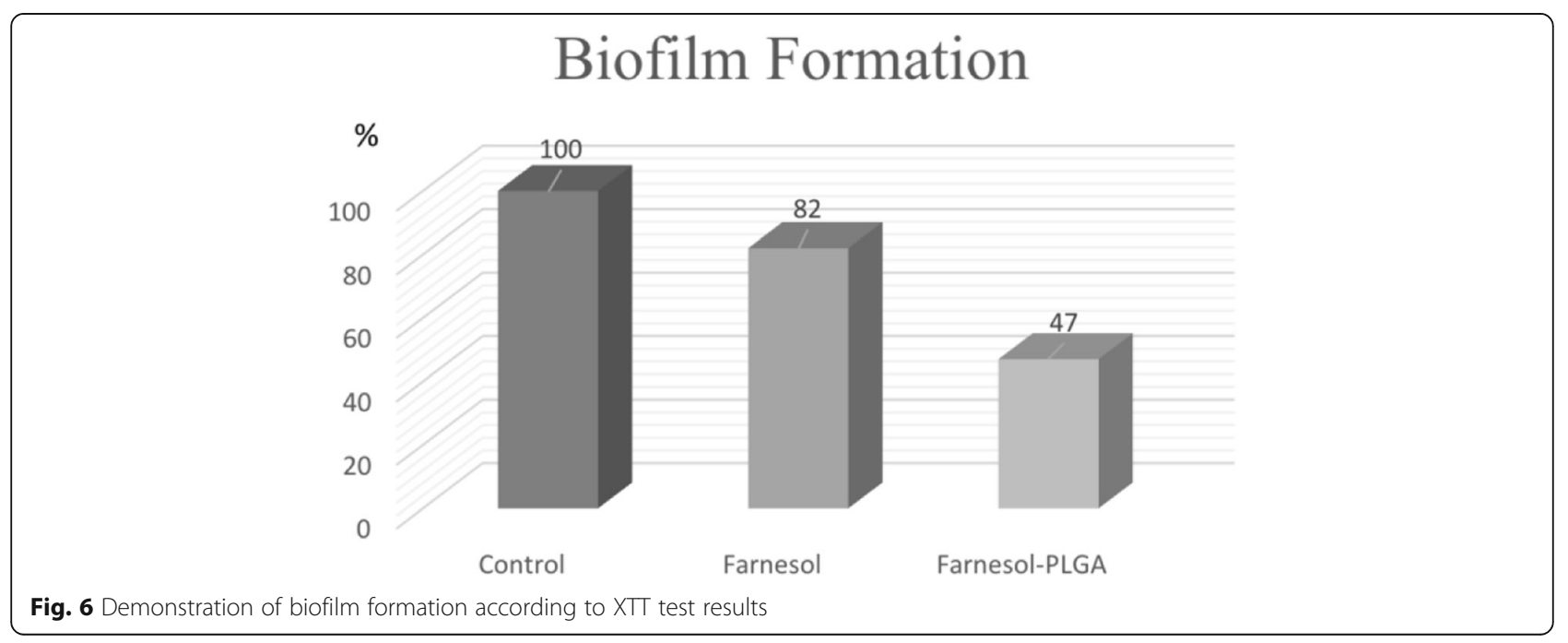




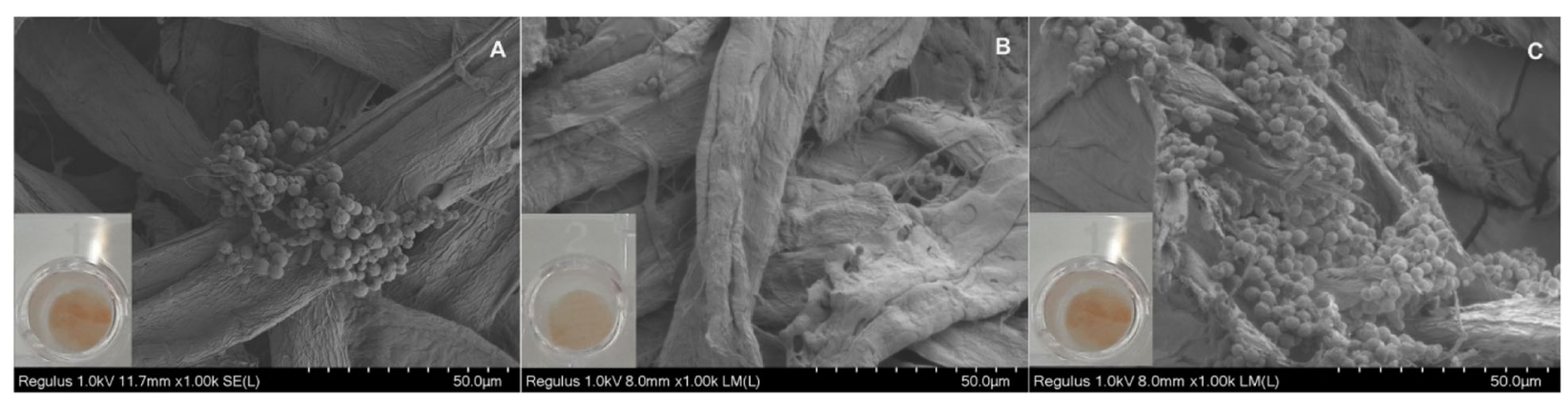

Fig. 7 SEM images of the C. albicans cells treated with farnesol alone and F-PLGA NPS on biofilm formation

disrupted or the adhesion of microorganisms on the surface may be limited (Sebaa et al. 2019). On the other hand, farnesol, a hydrophobic drug, has been reported to have antimicrobial effects against certain pathogens such as $S$. mutans. However, due to the limits of solubility associated with hydrophobicity and poor biofilm penetration, the use of high concentrations of drug is required in biofilm treatment. Studies have shown that when polymeric nanoparticles are used to increase the efficacy of farnesol, its effectiveness on oral biofilm increases (Sims et al. 2019). On the other hand, the interactions between biofilm and the active substance used are the most important factors in determining the effectiveness of the treatment. The EPS structure in the biofilm is heterogeneous and limits the penetration of antimicrobials (Wang et al., 2016). Electrostatic, hydrophobic, and other non-covalent interactions of active substances that interact with both microorganism and EPS structure are important in antibiotic film strategies.

Ability of farnesol to prevent yeast-hyphae transmission is highly promising both in the control of infection and in optimizing the antifungal effect of other drugs (Katragkou et al, 2014). Arasoğlu et al. investigated the antimicrobial effects of quercetin-loaded PLGA nanoparticles on four different bacterial isolates. In the study, the efficacy of bacterial isolates showed significant differences, and authors have reported that this may be due to different bacterial cell wall structures. Both quercetin and quercetin-laden NPs have been reported to be effective only on gram-positive bacteria, and the complex cell wall structure in gram-negatives constitutes a barrier for bacteria (Arasoglu et al., 2017). Costa et al. (2019) investigated the effects of farnesol and miconazole coencapsulated in biodegradable and biocompatible chitosan nanoparticles on the vulvovaginal candidiasis murine model. The authors reported that chitosan nanoparticles containing farnesol and miconazole are effective in inhibiting fungal growth. In addition, it was reported that farnesol-containing chitosan nanoparticles can reduce the pathogenicity of infection due to lack of inflammation (Costa et al. 2019). Esfandyari-Manesh et al. reported that it is very advantageous to use nanoparticles to arrest hydrophobic compounds with antimicrobial properties. These advantages include increased hydrophilicity, sustained release, and better penetration characteristics (Esfandyari-Manesh et al. 2013).

In our study, F-PLGA NPs were synthesized, characterized, and investigated for antimicrobial and antimicrobial properties on $C$. albicans. The synthesized F-PLGA NPs were approximately $140 \mathrm{~nm}$ in size, with a PDI of 0.463 and a positive zeta potential (658.5). Since the samples were prepared in aqueous solution in the zeta potential test, the nanoparticles were observed in larger size, whereas the dried samples were examined in TEM, so that the sample sizes were smaller. Roger et al. reported that nanoparticles with positive zeta potential between 50 and $300 \mathrm{~nm}$ are more effective especially for mucosal applications (Roger et al., 2010). In our study, NP dimensions were found to be in this range and zeta potential was positive and quite high. According to our ${ }^{1} \mathrm{H}$ NMR data, peaks of farnesol molecule were contained with peaks of PVA molecule in the 1-2 ppm range. On the other hand, all of the proton peaks of farnesol molecule except for $\mathrm{OH}$ group proton were observed in the spectrum of farnesol-loaded PLGA nanoparticles. It was concluded that in particular, the PLGA nanoparticle has chemically interacted with the $\mathrm{OH}$ functional group of the farnesol molecule.

In the present study, when ultrastructural effects of farnesol on planktonic Candida cells were examined by TEM, normal round-oval morphology was preserved in most of the cells, but few cells had wall and membrane damage and cytoplasm density in electrons. In the cells treated with F-PLGA NPs, the damaged findings were more increased, the number and volume of vacuoles increased, and deterioration in cell morphology and cell wall membrane expansion were observed. Decanis et al. investigated the effects of exogenous farnesol at different concentrations $(10,100,300 \mu \mathrm{M})$ on a $C$. albicans strain that does not produce endogenous farnesol. It has been shown that farnesol causes changes in cell wall shape in 
TEM findings. Also, a visible disconnection was detected between the cell wall and the cytoplasm. In addition, the vacuoles were observed in the cytoplasm (Décanis et al. 2011). Similar findings were found in also our study, but the ultrastructural damaging effects of F-PLGA NPs on the cell were found to be greater than farnesol alone. On the other hand, SEM micrographs obtained as a result of treatment of farnesol at different concentrations indicate deterioration in the external morphology of the Candida cells. The researchers stated that this situation indicates a decrease in cytosolic volume (Décanis et al. 2011)

In our study, farnesol and F-PLGA NPs were applied to Candida cells before biofilm formation and prebiofilm activities were investigated in both XTT and SEM analyses. Both analyses demonstrated the suppressive and reducing effects of F-PLGA NPs on biofilm formation, which were more potent than farnesol. In the literature, it is reported that farnesol prevents hyphal formation and biofilm development. On the contrary, tyrosol, another quorum-sensing molecule, stimulates hyphal formation. In the study of Alem et al., farnesol was added at three different concentrations $(50 \mu \mathrm{M}, 100 \mu \mathrm{M}$, and 1 $\mathrm{mM}$ ) and at different stages of the formation of C. albicans biofilm. Researchers have reported that early stages are sensitive to farnesol and these findings support our results (Alem et al. 2006). Katragkou et al. investigated the effects of combined use of farnesol with micafungin, fluconazole, and amphotericin B on C. albicans biofilms. Farnesol showed a synergistic or additive effect with these antifungals and structural changes were observed in the biofilm. The maximum combined effect was dependent on farnesol concentration (Katragkou et al., 2015). Chen et al. reported that the resistance of C. albicans biofilms to antifungals is associated with the enzymes CYR1 and PDE2, which are responsible for the synthesis and degradation of the cyclic AMP signaling pathway, and that farnesol reduces the antifungal resistance of C. albicans biofilms (Chen et al. 2018).

\section{Conclusion}

As a result, farnesol loaded to PLGA nanoparticles successfully by emulsion evaporation method. Our results demonstrate promising potential inhibitory effects of FPLGA nanoparticles when applied to both planktonic Candida cells and biofilm formation. Especially in FPLGA, a much smaller amount $22.5 \%$ of the total volume) of active substance was used compared to the use of farnesol alone and a similar effect to the efficacy of farnesol was observed. Inhibiting effects of hydrophobic molecule farnesol on mature Candida biofilms have also been reported, but nanoencapsulation systems can increase the effectiveness and potential of this interesting component in terms of their small size and large penetration areas. With detailed studies, it will be possible to obtain data that can shed light on the use of this molecule which regulates an important virulence factor such as yeast-hyphae transition. Further research is needed on the synergistic applications of the antifungals available with farnesol or on their effects when used with different biocompatible carriers.

\section{Abbreviations}

PLGA: Poly(DL-lactide-co-glycolide); F-PLGA Nps: Farnesol-loaded poly(DLlactide-co-glycolide) nanoparticles; XTT: (Sodium 3'-[1-[(phenylamino)carbony]-3,4-tetrazolium]-bis(4-methoxy-6-nitro)benzene-sulfonic acid hydrate); PBS: Phosphate-buffered saline; CYR1: The adenylate cyclase enzyme polypeptide; PDE2: Phosphodiesterase 2; AMP: Adenosine monophosphate

\section{Acknowledgements}

At the time of the study, all analyses were performed at Eskisehir Osmangazi University Central Research Laboratory Application and Research Center (ARUM), and I would like to thank the personnel from ARUM.

\section{Author's contributions}

The author read and approved the final manuscript.

Funding

Not applicable

Availability of data and materials

Not applicable

\section{Competing interests}

The author declares no conflict of interest.

Received: 6 January 2020 Accepted: 22 September 2020

Published online: 03 October 2020

\section{References}

Alem MA, Oteef MD, Flowers TH, Douglas L. Production of tyrosol by Candida albicans biofilms and its role in quorum sensing and biofilm development. Eukaryot Cell. 2006;5(10):1770-9.

Arasoglu T, Derman S, Mansuroglu B, Yelkenci G, Kocyigit B, Gumus B, Kocacaliskan I. Synthesis, characterization and antibacterial activity of juglone encapsulated PLGA nanoparticles. J Appl Microbiol. 2017;123(6):1407-19.

Cao Y-Y, Cao Y-B, Xu Z, Ying K, Li Y, Xie Y, Zhu Z-Y, Chen W-S, Jiang Y-Y. cDNA microarray analysis of differential gene expression in Candida albicans biofilm exposed to farnesol. Antimicrob Agents Chemother. 2005:49(2):584-9.

Chen S, Xia J, Li C, Zuo L, Wei X. The possible molecular mechanisms of farnesol on the antifungal resistance of $\mathrm{C}$. albicans biofilms: the regulation of CYR1 and PDE2. BMC Microbiol. 2018;18(1):203.

Costa A, Araujo D, Cabral M, Brito I, de Menezes LL, Pereira M, Amaral A. Development, characterization, and in vitro-in vivo evaluation of polymeric nanoparticles containing miconazole and farnesol for treatment of vulvovaginal candidiasis. Med Mycol. 2019;57(1):52-62.

Dag I, Acar M, Sakallioglu O, Catli T, San T, Cingi C. Influence of surface properties of Merocel (polyvinyl acetal) and silicone nasal splints on biofilm formation. Eur Arch Otorhinolaryngol. 2014;271(6):1519-24.

Décanis N, Tazi N, Correia A, Vilanova M, Rouabhia M. Farnesol, a fungal quorumsensing molecule triggers Candida albicans morphological changes by downregulating the expression of different secreted aspartyl proteinase genes. Open Microbiol J. 2011;5:119.

Esfandyari-Manesh M, Ghaedi Z, Asemi M, Khanavi M, Manayi A, Jamalifar H, Atyabi F, Dinarvand R. Study of antimicrobial activity of anethole and carvone loaded PLGA nanoparticles. J Pharm Res. 2013;7(4):290-5.

Gomes C, Moreira RG, Castell-Perez E. Poly (DL-lactide-co-glycolide)(PLGA) nanoparticles with entrapped trans-cinnamaldehyde and eugenol for antimicrobial delivery applications. J Food Sci. 2011;76(2):N16-24.

Hornby JM, Jensen EC, Lisec AD, Tasto JJ, Jahnke B, Shoemaker R, Dussault P, Nickerson KW. Quorum sensing in the dimorphic fungus Candida albicans is mediated by farnesol. Appl Environ Microbiol. 2001;67(7):2982-92. 
Ilknur, D., Yasemin, O., Nuri, K. Effect of disinfectants on biofilm development by five species of Candida. African Journal of Microbiology Research. 2012;6(10): 2380-2386.

Jeon JG, Pandit S, Xiao J, Gregoire S, Falsetta ML, Klein Ml, Koo H. Influences of trans-trans farnesol, a membrane-targeting sesquiterpenoid, on Streptococcus mutans physiology and survival within mixed-species oral biofilms. Int J Oral Sci. 2011;3(2):98.

Katragkou A, McCarthy M, Alexander EL, Antachopoulos C, Meletiadis J, Jabra-Rizk MA, Petraitis V, Roilides E, Walsh TJ. In vitro interactions between farnesol and fluconazole, amphotericin B or micafungin against Candida albicans biofilms. J Antimicrob Chemother. 2014;70(2):470-8.

Katragkou A, McCarthy M, Alexander EL, Antachopoulos C, Meletiadis J, Jabra-Rizk MA, Walsh TJ. In vitro interactions between farnesol and fluconazole, amphotericin B or micafungin against Candida albicans biofilms. J Antimicrob Chemother. 2015:70(2):470-8.

Laihadı FM, Supriyadi H, Hermanto E, Elidasari M, Soemartono GHH. Case report: Fungal infections in the normal gingival mucosa affecting oral surgery; 2017.

Landis RF, Gupta A, Lee Y-W, Wang L-S, Golba B, Couillaud B, Ridolfo R, Das R, Rotello VM. Cross-linked polymer-stabilized nanocomposites for the treatment of bacterial biofilms. ACS Nano. 2016;11(1):946-52.

Lindsay AK, Deveau A, Piispanen AE, Hogan DA. Farnesol and cyclic AMP signaling effects on the hypha-to-yeast transition in Candida albicans, Eukaryot Cell. 2012;11(10):1219-25.

Nickerson KW, Atkin AL, Hornby JM. Quorum sensing in dimorphic fungi: farnesol and beyond. Appl Environ Microbiol. 2006;72(6):3805-13

Oz Y, Kiremitci A, Dag I, Metintas S, Kiraz N. Postantifungal effect of the combination of caspofungin with voriconazole and amphotericin B against clinical Candida krusei isolates. Med Mycol. 2013;51(1):60-5.

Ozturk BY, Gursu BY, Dag I. Antibiofilm and antimicrobial activities of green synthesized silver nanoparticles using marine red algae Gelidium corneum. Process Biochem. 2020;89:208-19.

Roger E, Lagarce F, Garcion E, Benoit JP. Biopharmaceutical parameters to consider in order to alter the fate of nanocarriers after oral delivery. Nanomedicine. 2010;5(2):287-306.

Rajendran R, Sherry L, Deshpande A, Johnson EM, Hanson MF, Williams C, Munro CA, Jones BL, Ramage G. A prospective surveillance study of candidaemia: epidemiology, risk factors, antifungal treatment and outcome in hospitalized patients. Front Microbiol. 2016;7:915.

Ramage G, Walle KV, Wickes BL, Lopez-Ribot JL. Characteristics of biofilm formation by Candida albicans. Rev Iberoam Micol. 2001;18(4):163-70

Rowat AC, Keller D, Ipsen JH. Effects of farnesol on the physical properties of DMPC membranes. Biochimica et Biophysica Acta (BBA)-Biomembranes. 2005;1713(1):29-39.

Sebaa S, Boucherit-Otmani Z, Courtois P. Effects of tyrosol and farnesol on Candida albicans biofilm. Mol Med Rep. 2019;19(4):3201-9.

Sims KR, Liu Y, Hwang G, Jung HI, Koo H, Benoit DS. Enhanced design and formulation of nanoparticles for anti-biofilm drug delivery. Nanoscale. 2019; 11(1):219-36

Wang, L., Li, Y., Wang, L., Zhang, H., Zhu, M., Zhang, P., Zhu, X. Extracellular polymeric substances affect the responses of multi-species biofilms in the presence of sulfamethizole. Environ. Pollut. 2018;235:283-292.

Wayne P: Reference method for broth dilution antifungal susceptibility testing of yeasts, approved standard. CLSI document M27-A2 2002.

\section{Publisher's Note}

Springer Nature remains neutral with regard to jurisdictional claims in published maps and institutional affiliations.

\section{Submit your manuscript to a SpringerOpen ${ }^{\circ}$ journal and benefit from:}

- Convenient online submission

- Rigorous peer review

- Open access: articles freely available online

High visibility within the field

- Retaining the copyright to your article

Submit your next manuscript at $\boldsymbol{\nabla}$ springeropen.com 